\title{
Validation of Postinduction Curie Scores in High-Risk Neuroblastoma: A Children's Oncology Group and SIOPEN Group Report on SIOPEN/HR-NBL1
}

\author{
Gregory A. Yanik ${ }^{1}$, Marguerite T. Parisi ${ }^{2,3}$, Arlene Naranjo ${ }^{4}$, Helen $\mathrm{Nadel}^{5}$, Michael J. Gelfand ${ }^{6}$, Julie R. Park ${ }^{3}$, \\ Ruth L. Ladenstein ${ }^{7}$, Ulrike Poetschger ${ }^{8}$, Ariane Boubaker ${ }^{9}$, Dominique Valteau-Couanet ${ }^{10}$, Bieke Lambert ${ }^{11}$, \\ Maria-Rita Castellani ${ }^{12}$, Zvi Bar-Sever ${ }^{13}$, Aurore Oudoux ${ }^{14}$, Anna Kaminska ${ }^{15}$, Susan G. Kreissman ${ }^{16}$, \\ Barry L. Shulkin ${ }^{17}$, and Katherine K. Matthay ${ }^{18}$
}

\begin{abstract}
${ }^{1}$ Department of Pediatrics and Communicable Diseases, University of Michigan Medical Center, Ann Arbor, Michigan; ${ }^{2}$ Department of Radiology, University of Washington School of Medicine/Seattle Children's Hospital, Seattle, Washington; ${ }^{3}$ Department of Pediatrics, University of Washington School of Medicine/Seattle Children's Hospital, Seattle, Washington; ${ }^{4}$ Children's Oncology Group Statistics and Data Center, University of Florida, Gainesville, Florida; 5 Department of Radiology, BC Children's Hospital, Vancouver, British Columbia, Canada; ${ }^{6}$ Department of Radiology, Cincinnati Children's Hospital Medical Center, Cincinnati, Ohio; ${ }^{7}$ Department of Pediatrics, St. Anna Children's Hospital, Vienna, Austria; ${ }^{8}$ Department of Statistics, St. Anna Children's Cancer Research Institute, Vienna, Austria; ${ }^{9}$ Institute of Radiology, Clinique de La Source, Lausanne, Switzerland; ${ }^{10}$ Department of Pediatric and Adolescent Oncology, Gustave Roussy Institute, Universite Paris-Sud, Villejuif, France; ${ }^{11}$ Radiology and Nuclear Medicine, Ghent University, Ghent, Belgium; ${ }^{12}$ Nuclear Medicine Division, Istituto Nazionale Tumori di Milano, Milan, Italy; ${ }^{13}$ Schneider Children's Medical Center of Israel, Petah-Tivka, Israel; ${ }^{14}$ Centre Oscar-Lambret, Lille, France; ${ }^{15}$ Children's Memorial Health Institute, Warsaw, Poland; ${ }^{16}$ Department of Pediatrics, Duke University Medical Center, Durham, North Carolina; ${ }^{17}$ Department of Diagnostic Imaging, St. Jude Children's Research Hospital, Memphis, Tennessee; and ${ }^{18}$ Department of Pediatrics, University of California San Francisco School of Medicine, San Francisco, California
\end{abstract}

A semiquantitative ${ }^{123}$ |-metaiodobenzylguanidine (123|-MIBG) scoring method (the Curie score, or CS) was previously examined in the Children's Oncology Group (COG) high-risk neuroblastoma trial, COG A3973, with a postinduction CS of more than 2 being associated with poor event-free survival (EFS). The validation of the CS in an independent dataset, International Society of Paediatric Oncology European Neuroblastoma/High-Risk Neuroblastoma 1 (SIOPEN/ HR-NBL1), is now reported. Methods: A retrospective analysis of 123/-MIBG scans obtained from patients who had been prospectively enrolled in SIOPEN/HR-NBL1 was performed. All patients exhibited ${ }^{123}$ I-MIBG-avid, International Neuroblastoma Staging System stage 4 neuroblastoma. ${ }^{123}$ I-MIBG scans were evaluated at 2 time points, diagnosis $(n=345)$ and postinduction $(n=330)$, before consolidation myeloablative therapy. Scans of 10 anatomic regions were evaluated, with each region being scored $0-3$ on the basis of disease extent and a cumulative CS generated. Cut points for outcome analysis were identified by Youden methodology. CSs from patients enrolled in COG A3973 were used for comparison. Results: The optimal cut point for CS at diagnosis was 12 in SIOPEN/HR-NBL1, with a significant outcome difference by CS noted (5-y EFS, $43.0 \% \pm 5.7 \%$ [CS $\leq 12$ ] vs. $21.4 \% \pm 3.6 \%$ [CS $>12$ ], $P<0.0001$ ). The optimal CS cut point after induction was 2 in SIOPEN/HR-NBL1, with a postinduction CS of more than 2 being associated with an inferior outcome (5-y EFS, $39.2 \% \pm 4.7 \%$ [CS $\leq 2$ ] vs. $16.4 \% \pm 4.2 \%$ [CS $>2$ ], $P<0.0001$ ). The postinduction CS maintained independent statistical significance in Cox models when adjusted for the covariates of age and $M Y C N$ gene copy number. Conclusion: The prognostic

Received May 9, 2017; revision accepted Jul. 12, 2017.

For correspondence or reprints contact: Gregory A. Yanik, University of Michigan, 1500 E. Medical Center Dr., MPB D4216, Ann Arbor, MI 48109. E-mail: gyanik@umich.edu

Published online Sep. 8, 2017.

COPYRIGHT (C 2018 by the Society of Nuclear Medicine and Molecular Imaging. significance of postinduction CSs has now been validated in an independent cohort of patients (SIOPEN/HR-NBL1), with a postinduction CS of more than 2 being associated with an inferior outcome in 2 independent large, cooperative group trials.

Key Words: neuroblastoma; MIBG; Curie score

J Nucl Med 2018; 59:502-508

DOI: 10.2967/jnumed.117.195883

D

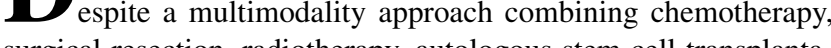
surgical resection, radiotherapy, autologous stem cell transplantation, and biotherapy, survival for high-risk neuroblastoma remains poor, with a 5-y event-free survival (EFS) of 30\%-49\% (1-3). The use of the anti-GD2 chimeric antibody (ch14.18, dinutuximab [Unituxin; United Therapeutics Corp.]) after transplantation in combination with granulocyte-macrophage colony-stimulating factor and intravenous interleukin-2 has led to improvements in EFS (4). For patients who develop relapsed disease, specifically those who relapse 6-18 mo from initial diagnosis, 5-y overall survival (OS) is less than $20 \%$ (5). The identification of prognostic markers of response and survival early in a patient's treatment may have a significant impact on therapy and outcome.

Metaiodobenzylguanidine (MIBG) is a guanethidine analog that has been used as a diagnostic imaging agent for neuroblastoma for over 30 y (6-9). Uptake is well described in marrow, osseous sites, and soft-tissue sites of disease. In 1995, a MIBG scoring system was developed to semiquantify the extent of MIBG uptake within individual patients and to serve as an imaging biomarker for outcome prediction (10). The role of semiquantitative MIBG scoring 


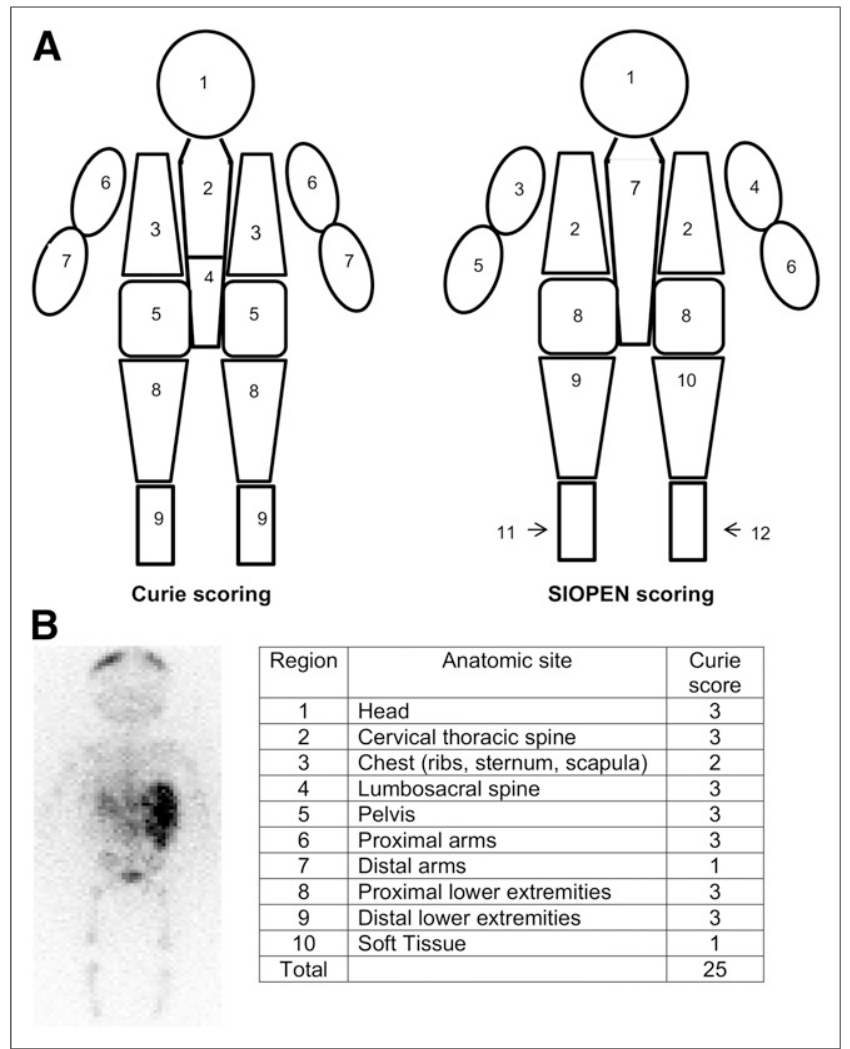

FIGURE 1. (A) Anatomic regions for CS and SIOPEN score. Body is divided into 9 (CS) or 12 (SIOPEN score) skeletal regions, with CS adding 10th (soft-tissue) region. (B) Example of CS of individual patient: diffuse uptake (CS, 3) is noted in head, cervical-thoracic spine, lumbar-sacral spine, pelvis, proximal arms, proximal lower extremities, distal lower extremities, and chest (CS, 2). There is one ${ }^{123} \mid-M I B G-a v i d$ soft-tissue site, involving $<50 \%$ abdomen (CS, 1). Total CS $=25$.

as a prognostic indicator for high-risk neuroblastoma has now been reported in both institutional and cooperative group trials, including trials within the Children's Oncology Group (COG) and the International Society of Paediatric Oncology European Neuroblastoma (SIOPEN) Research Network (10-20). In particular, the presence of MIBG-avid disease after induction has correlated with extremely poor EFS and OS after consolidation therapy, including high-dose chemotherapy with autologous stem cell transplantation (11,14-20). Likewise, MIBG scores at diagnosis have been prognostic in SIOPEN high-risk neuroblastoma studies (20). Scan type, ${ }^{131}$ I versus ${ }^{123}$ I MIBG, has not affected outcome predictions, either at diagnosis or after induction (21).

Two MIBG scoring methods are now commonly used, the Curie score (CS) and the SIOPEN score $(14,19,20)$. The 2 scoring methods subdivide the skeleton into 9 (CS) or 12 (SIOPEN score) regions (Fig. 1), with CS adding a tenth region for evaluating softtissue disease in both primary and metastatic sites. Scores from each region (ranging from 0 to 3 ) are summed to determine a cumulative CS (or SIOPEN score). The CS method has been adapted for use in COG trials, including the high-risk neuroblastoma trial, COG A3973 (22). The SIOPEN score has been adapted for use in SIOPEN high-risk neuroblastoma trials, including the recent International Society of Pediatric Oncology European Neuroblastoma/ High-Risk Neuroblastoma 1 (SIOPEN/HR-NBL1).
For patients treated in COG A3973, a postinduction CS of more than 2 was associated with an inferior outcome, when compared with patients with a CS of 2 or less after induction (5-y EFS, $10.5 \% \pm 10.0 \%$ vs. $42.0 \% \pm 5.8 \%, P<0.0001$ ) (19). Furthermore, a postinduction CS of more than 2 identified patients at high risk for an event in COG A3973, independent of other known prognostic factors, including age, MYCN (v-myc avian myelocytomatosis viral oncogene neuroblastoma derived homolog) status, ploidy, and histologic grade (19).

We now report the prognostic value of the CS of ${ }^{123}$ I-MIBG scans at diagnosis and at the end of induction for patients treated in the European high-risk trial (SIOPEN/HR-NBL1), with subsequent comparisons to an independent cohort of patients treated in the COG high-risk neuroblastoma protocol, COG A3973. The current study validates the role of CS as a prognostic marker of response and survival in a large, independent dataset of patients with ${ }^{123} \mathrm{I}-$ MIBG-avid, stage 4, newly diagnosed high-risk neuroblastoma.

\section{MATERIALS AND METHODS}

\section{Patient Population}

Three hundred forty-five patients with newly diagnosed stage 4, highrisk neuroblastoma (per International Neuroblastoma Staging System) (23) who were enrolled in SIOPEN/HR-NBL1 and had ${ }^{123}$ I-MIBG-avid disease at diagnosis were examined (Table 1; Fig. 2). Determining the prognostic value of ${ }^{123}$ I-MIBG scoring was a stated aim of SIOPEN/HR-NBL1, with scoring performed retrospectively, examining ${ }^{123} \mathrm{I}-\mathrm{MIBG}$ scans obtained at diagnosis $(n=345)$ and after induction $(n=330)$.

\section{Treatment}

SIOPEN/HR-NBL1 (NCT00030719) is a randomized, multicenter study for patients less than 21 y old with biopsy-proven high-risk neuroblastoma (patient enrollment, 2002-2010) (3). Inclusion criteria were stage 2-4 disease with $M Y C N$ amplification (any age) or stage 4

TABLE 1

Characteristics at Diagnosis in Patients with ${ }^{123}$-MIBGavid, Stage 4 Disease

\begin{tabular}{|cc}
\hline Characteristic & $n$ \\
\hline Total patients & 345 \\
\hline Sex & $212(61 \%)$ \\
\hline Male & $133(39 \%)$ \\
\hline Female & \\
\hline Age & $2.9(\mathrm{range}, 0.2 \%-16.1 \%)$ \\
\hline Median age & $48(14 \%)$ \\
\hline Age $<18$ mo & $297(86 \%)$ \\
\hline Age $\geq 18$ mo & \\
\hline MYCN status & $122(35 \%)$ \\
\hline Amplified & $182(53 \%)$ \\
\hline Nonamplified & $41(12 \%)$ \\
\hline Unknown & $261(76 \%)$ \\
\hline ASCT & $23(7 \%)$ \\
\hline Chimeric antibody &
\end{tabular}

ASCT $=$ autologous stem cell transplantation, which was performed if patient was in CR/PR with $\leq 3$ 123/-MIBG-avid sites and $>50 \%$ reduction of ${ }^{123}$ /-MIBG-avid osseous lesions. 


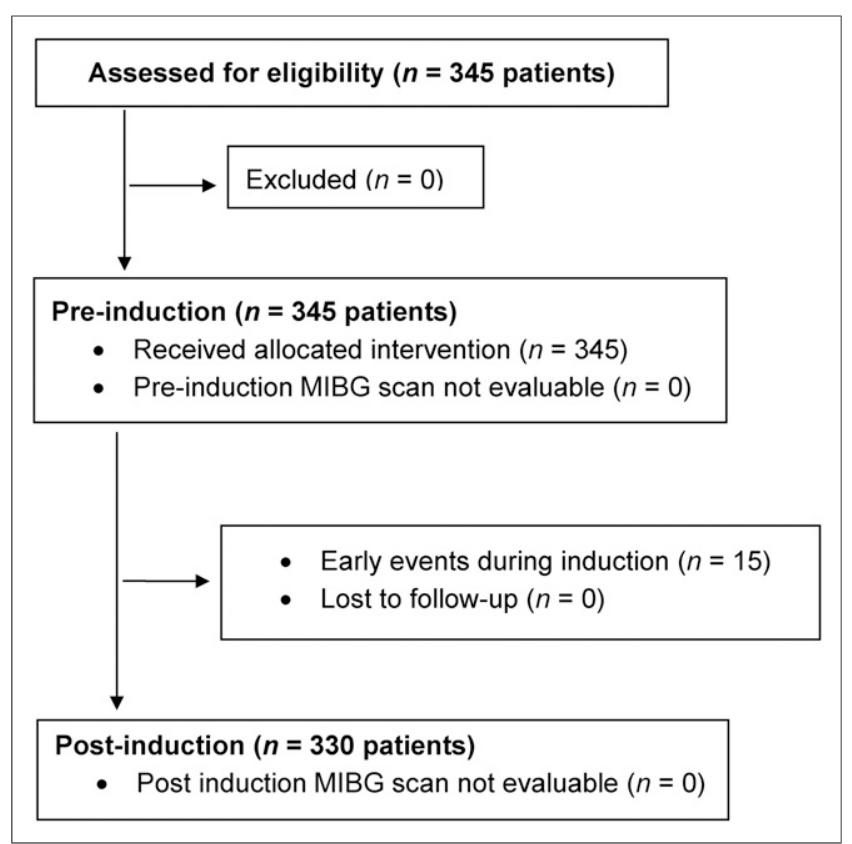

FIGURE 2. CONSORT (consolidated standards of reporting trials) diagram: CS analysis of SIOPEN/HR-NBL1.

disease in patients older than 1 y. Only the cohort with ${ }^{123}$ I-MIBG-avid stage 4 disease was used for the current evaluation. Therapy on SIOPEN/HR-NBL1 included 8 cycles of an intensively timed induction regimen (Rapid COJEC, which consisted of repetitive courses of vincristine-carboplatin-etoposide [cycle A], vincristine-cisplatin [course $\mathrm{B}$ ], and vincristine-etoposide-cyclophosphamide [course C]), surgical resection of residual disease (after induction) followed by ${ }^{123}$ I-MIBG scanning, high-dose chemotherapy with autologous stem cell transplantation, local radiotherapy, and subsequent maintenance therapy with isotretinoin, as previously published (Fig. 3) (2,3,24,25). Immunotherapy with an anti-GD2 monoclonal antibody (ch14.18) with or without subcutaneous interleukin-2 was given to patients enrolled from 2007 onward. Only patients in complete (CR) or partial remission (PR) with no more than $3{ }^{123} \mathrm{I}$-MIBG-avid sites on completion of induction and more than a $50 \%$ reduction in ${ }^{123}$ I-MIBG-avid osseous lesions were eligible to proceed to autologous stem cell transplantation. Patients who failed to meet these criteria received 2 additional courses of topotecan, vincristine, and doxorubicin before high-dose chemotherapy, and surgery was postponed until a better metastatic response was achieved. Transplantation was performed using either a busulfanmelphalan or a carboplatin-etoposide-melphalan conditioning regimen. Written informed consent (approved by local ethics boards) was obtained from patients (or legal guardians) before entry into SIOPEN/HR-NBL1. The trial was registered at Clinicaltrials.gov (NCT00030719, SIOPEurope-HR-NBL-1, CDR0000069191, ESIOP, EU-20148) and at EudraCT (2006-001489-17).

\section{CS Determinations}

CS was determined from diagnostic ${ }^{123} \mathrm{I}-\mathrm{MIBG}$ scans as previously reported $(14,19)$. Planar images were acquired $24 \mathrm{~h}$ after administration of ${ }^{123}$ I-MIBG, with scans obtained at diagnosis and after induction (before surgery). Patient scans were evaluated for ${ }^{123}$ I-MIBG avidity at 10 different sites (19). Skeletal sites were individually scored 0-3 ( 0 , no ${ }^{123}$ I-MIBG-avid lesions; 1 , one ${ }^{123}$ I-MIBG-avid lesion; 2 , more than one ${ }^{123}$ I-MIBG-avid lesion; and 3, ${ }^{123}$ I-MIBG uptake in $>50 \%$ of an individual skeletal site). Soft-tissue lesions were also scored 0-3 ( 0 , no ${ }^{123} \mathrm{I}-\mathrm{MIBG}$ involvement; 1 , one ${ }^{123} \mathrm{I}-\mathrm{MIBG}$-avid soft-tissue lesion;
2, more than one ${ }^{123}$ I-MIBG-avid soft-tissue lesion in one or more regions; and 3, ${ }^{123}$ I-MIBG avidity in a soft-tissue lesion that occupied $>50 \%$ of the chest or abdomen). Both the primary and the metastatic softtissue lesions were included to create a composite soft-tissue score. A patient's overall CS was calculated as the sum of scores over all 10 individual sites, with a maximum possible CS of 30 . The ${ }^{123} \mathrm{I}-\mathrm{MIBG}$ scans were centrally reviewed by 4 pediatric nuclear medicine physicians from the COG diagnostic imaging committee, with reviewers masked to clinical or radiographic reports. The reviewers scored each scan as a collective group, to establish a consensus CS for each case. Two of the 4 reviewers had previously reviewed all ${ }^{123}$ I-MIBG scans from COG A3973 (19).

\section{Statistical Analysis}

Patients were categorized by CS $(0$ vs. $>0$; $\leq$ optimum cutoff vs. $>$ optimum cutoff) and compared with respect to survival (EFS and OS) at each time point. The optimum cutoff was determined by maximizing the Youden index with respect to how well the CS differentiated patients who had and did not have an event. The Youden index is the maximum of [sensitivity + specificity -1 ] over all threshold values (0-30) of the CS $(26,27)$. Survival comparisons were drawn at each individual site by score ( $0 \mathrm{vs}$. $>0$ only). The percentage change in CS from diagnosis to postinduction scan for patients with both sets of ${ }^{123}$ I-MIBG scan readings was stratified on the basis of score reduction $(\geq 50 \%$ reduction vs. $<50 \%$, and $\geq 75 \%$ reduction vs. $<75 \%$ ), with survival compared between groups.

For EFS, time to event was defined as the time from diagnosis until the time of first occurrence of relapse, progressive disease, secondary malignancy, or death or until the time of last contact if no event occurred. Patients who were alive without an event were censored at the last known date of contact. For OS, death was the only event considered. After induction, time to event was calculated from the date of the postinduction ${ }^{123}$ I-MIBG scan. Patients who had an event between the date of diagnosis and the date of postinduction ${ }^{123} \mathrm{I}-\mathrm{MIBG}$ scan $(n=15)$ were considered to have gone off-study and hence were not included in the analysis after induction. Survival was analyzed using the methods of Kaplan and Meier, with standard errors per the methods of Peto et al. $(28,29)$. Survival curves were compared using a log-rank test. Cox proportional hazards models with the Efron model of handling tied events were fit to determine the CS cutoff at which the hazard ratio was maximized, as well as the prognostic strength for survival of the CS in the presence of age $(<18 \mathrm{mo}$ vs. $\geq 18 \mathrm{mo}$ ) and $M Y C N$ gene copy number (nonamplified vs. amplified). $P$ values of less than 0.05 were considered statistically significant.

\section{RESULTS}

Three hundred forty-five patients had ${ }^{123}$ I-MIBG scans available at diagnosis, with postinduction scans available in 330 patients and

\begin{tabular}{|c|c|c|c|c|c|c|c|c|c|c|c|}
\hline$\leftarrow$ & $\begin{array}{l}\text {-NBL } \\
\text { Ind }\end{array}$ & -1/SI & on (R & apid & $\mathrm{co}$ & EC) & $\longrightarrow$ & $\leftarrow$ & - Con & olidati & \\
\hline 1 & 2 & 3 & 4 & 5 & 6 & 7 & 8 & Surgery & Вмт & XRT & Isotretinoin \\
\hline \multicolumn{9}{|l|}{ MIBG scan } & \multicolumn{3}{|c|}{ BuMel vs CEM } \\
\hline \multicolumn{12}{|c|}{ COG A3973 } \\
\hline \multicolumn{12}{|c|}{$\longleftarrow$ Induction (Modified N7) $\longrightarrow$} \\
\hline 1 & 2 & & 3 & 4 & 5 & & Surgery & 6 & BMT & XRT & Isotretinoin \\
\hline MIBG scan & & & & & & & & MIBG scan & CEM & & \\
\hline
\end{tabular}

FIGURE 3. Schematic overview of SIOPEN/HR-NBL1 (2) and COG A3973 (22) therapy. Biotherapy $=$ isotretinoin \pm anti-GD2 chimeric antibody; $\mathrm{BMT}=$ bone marrow transplant; BuMel = busulfan-melphalan; $\mathrm{CEM}$ = carboplatin-etoposide-melphalan; XRT = radiotherapy. 


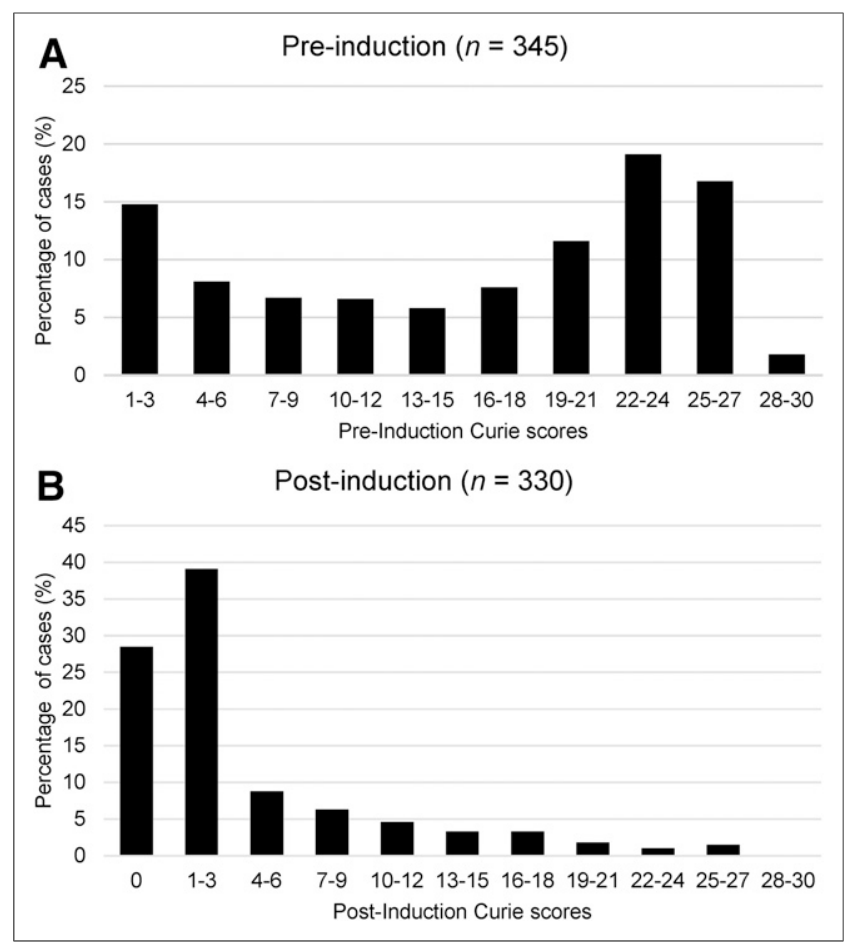

FIGURE 4. Distribution of CSs at (A) diagnosis, and (B) after induction.

both sets of images evaluable in 329 patients (Table 1). The median CS at diagnosis was 19 (range, 1-30), with 149 patients (43.2\%) exhibiting a CS of more than 20 at diagnosis (Fig. 4A). The median score after induction was 1.5 (range, 0-27), with only 14 patients (4.2\%) exhibiting a CS of more than 20 at that time point (Fig. 4B). Seventy-six percent of patients in the SIOPEN/ HR-NBL1 cohort underwent myeloablative therapy according to SIOPEN/HR-NBL1 guidelines.

\section{CS at Diagnosis and Outcome}

The 5-y EFS and OS for the 345 patients with ${ }^{123} \mathrm{I}-\mathrm{MIBG}$ scan reviews at diagnosis were $29.3 \% \pm 3.2 \%$ and $40.5 \% \pm 3.4 \%$, respectively. The optimal cut point for analyses, as determined by the Youden index, was a CS of 12. Five-year EFS was $43.0 \% \pm 5.7 \%$ for patients with a CS of no more than $12(n=$ 126 ), versus $21.4 \% \pm 3.6 \%$ for those with a score of more than 12 $(n=219)$ at diagnosis, $P<0.001$ (Table 2; Fig. 5). Differences in OS were likewise noted, with a 5 -y OS of $53.9 \% \pm 5.6 \%$ versus $32.7 \% \pm 4.0 \%$ for patients with a CS of 12 or less versus more than 12 , respectively $(P=0.001)$. The Cox proportional hazards model found CS to be predictive of EFS and OS after adjusting for MYCN and age. Patients with a CS of more than 12 had an increased risk of an event and death of 1.802 and 1.765 , respectively.

The median soft-tissue score (region 10) at diagnosis was 1 (range, 0-3), with 34 patients $(9.9 \%)$ exhibiting no ${ }^{123} \mathrm{I}-\mathrm{MIBG}-$ avid soft-tissue disease at diagnosis and 35 patients (10.1\%) exhibiting avidity in more than 1 soft-tissue site. The presence of ${ }^{123}$ I-MIBG-avid soft-tissue disease at diagnosis did not affect EFS, with 5 -y EFS of $29.2 \% \pm 3.3 \%$ versus $29.4 \% \pm 10.1 \%$ for patients with versus without ${ }^{123} \mathrm{I}-\mathrm{MIBG}$-avid soft-tissue disease, respectively, at that time point $(P=0.64)$.

MYCN status was available in $304(88.1 \%)$ of the 345 patients, with $M Y C N$-amplified disease present in 122 cases and $M Y C N$ nonamplified disease present in 182. Patients with a CS of more than 12 at diagnosis had inferior survival, independent of MYCN status. For patients with $M Y C N$-amplified disease, a significant outcome difference existed by CS at diagnosis, with 5-y EFS of $39.3 \% \pm$ $7.9 \%(\mathrm{CS} \leq 12)$ versus $19.1 \% \pm 8.6 \%(\mathrm{CS}>12)(P=0.013)$. For patients with $M Y C N$-nonamplified disease, a significant outcome difference likewise existed by CS, with 5-y EFS of $42.8 \% \pm$ $9.0 \%(\mathrm{CS} \leq 12)$ versus $23.4 \% \pm 4.5 \%(\mathrm{CS}>12)(P=0.008)$.

\section{Postinduction CS and Outcome}

The 5-y EFS and OS for the 330 patients with ${ }^{123}$ I-MIBG scans after induction were $30.1 \% \pm 3.4 \%$ and $40.6 \% \pm 3.5 \%$, respectively. The optimum cut point after induction was a CS of 2 . In addition, Cox proportional hazards models indicated that a CS of 2 after induction corresponded to the largest significant hazard ratio (1.710; 95\% confidence interval, 1.314-2.226) when compared with other potential cut points. Patients with a CS of 2 or less $(n=198,60 \%)$ after induction had significantly better 5-y EFS than patients with a CS of more than $2(n=132)(39.2 \% \pm 4.7 \%$

TABLE 2

EFS and OS by CS at Diagnosis and After Induction, and Percentage Reduction in CS

\begin{tabular}{|c|c|c|c|c|c|}
\hline CS & $n$ & $5-y$ EFS \pm SE (\%) & EFS $P$ & $5-y$ OS \pm SE (\%) & OS $P$ \\
\hline \multicolumn{6}{|c|}{ At diagnosis } \\
\hline$\leq 12$ & $126(37 \%)$ & $43.0 \pm 5.7$ & & $53.9 \pm 5.6$ & \\
\hline 12 & $219(63 \%)$ & $21.4 \pm 3.6$ & $<0.001$ & $32.7 \pm 4.0$ & 0.001 \\
\hline \multicolumn{6}{|c|}{ After induction } \\
\hline$\leq 2$ & $198(60 \%)$ & $39.2 \pm 4.7$ & & $48.0 \pm 4.7$ & \\
\hline 2 & 132 (40\%) & $16.4 \pm 4.2$ & $<0.001$ & $29.5 \pm 5.1$ & $<0.001$ \\
\hline \multicolumn{6}{|l|}{ Reduction } \\
\hline$\geq 50 \%$ & $251(76 \%)$ & $31.8 \pm 3.9$ & & $40.6 \pm 4.0$ & \\
\hline $50 \%$ & $78(24 \%)$ & $25.3 \pm 6.9$ & 0.65 & $41.6 \pm 7.5$ & 0.91 \\
\hline$\geq 75 \%$ & $204(62 \%)$ & $32.3 \pm 4.4$ & & $41.2 \pm 4.5$ & \\
\hline $75 \%$ & $125(38 \%)$ & $26.9 \pm 5.3$ & 0.18 & $40.2 \pm 5.8$ & 0.39 \\
\hline
\end{tabular}




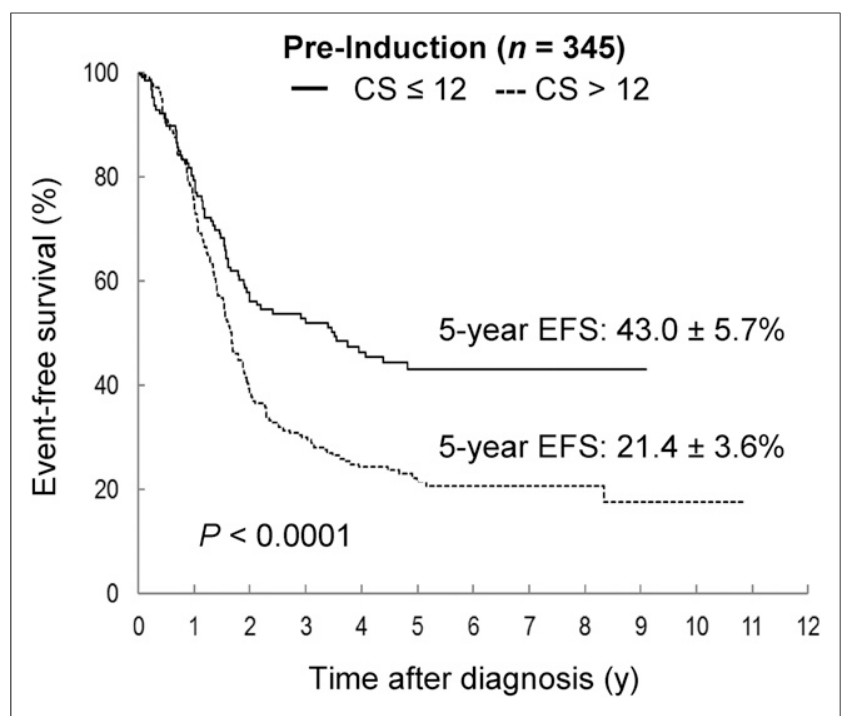

FIGURE 5. EFS by CS at diagnosis, using optimal cut point of 12: time to event starting from date of corresponding ${ }^{123}$ |-MIBG scan.

vs. $16.4 \% \pm 4.2 \%, P<0.001$ [Table 2; Fig. 6]). Likewise, there was a significant improvement in 5-y OS for patients with a CS of 2 or less after induction versus a CS of more than $2(48.0 \% \pm$ $4.7 \%$ vs. $29.5 \% \pm 5.1 \%, P<0.001)$. Outcome comparisons with a cutoff of 0 were also performed, yielding statistically significant differences for EFS but not for OS.

The median postinduction soft-tissue score was 1 (range, 0-3), with 137 patients $(41.5 \%)$ exhibiting no ${ }^{123}$ I-MIBG-avid soft-tissue disease at that time point. Improved 5-y EFS was noted in patients without ${ }^{123}$ I-MIBG-avid soft-tissue disease after induction $(37.8 \% \pm$ $6.1 \%$ [CS, 0$]$ vs. $25.2 \% \pm 3.9 \%$ [CS, $>0$ ], $P=0.04)$. When softtissue scores (region 10) were excluded from the analysis, the optimum cut point was a CS of 0 after induction (5-y EFS, $41.1 \% \pm$ $4.9 \%$ vs. $17.7 \% \pm 4.5 \%, \mathrm{CS}=0$ vs. $>0$ ).

The Cox models showed that CS was predictive of EFS and OS after adjusting for $M Y C N$ and age. Patients with a CS of more than 2 had an increased risk of an event and death of 1.791 and 1.769, respectively.

MYCN status was available in 290 of 330 patients with a postinduction CS. Patients with a CS of more than 2 after induction had inferior survival, independent of $M Y C N$ status. For patients with $M Y C N$-amplified disease, a significant outcome difference existed by postinduction CS, with a 5-y EFS of $38.0 \% \pm 7.0 \%$ $(\mathrm{CS} \leq 2)$ versus $13.8 \% \pm 12.8 \%(\mathrm{CS}>2)(P=0.001)$. For patients with $M Y C N$-nonamplified disease, a significant outcome difference likewise existed by postinduction CS, with 5-y EFS of $38.4 \% \pm 6.7 \%(\mathrm{CS} \leq 2)$ versus $18.7 \% \pm 5.3 \%(\mathrm{CS}>2)(P=$ 0.01). Outcome comparisons were not statistically significant using a cut point of 0 for $M Y C N$-amplified or -nonamplified tumors.

\section{Relative Scores: Change in CS from Diagnosis to Postinduction Time Points}

None of the outcome comparisons by percentage reduction in CS were statistically significant (Table 2). Specifically, no differences in survival were noted in patients with a $50 \%$ or greater reduction in CS versus a reduction of less than $50 \%$ between diagnosis and the postinduction time point. Likewise, no outcome differences were noted in patients with a $75 \%$ or greater reduction in score (vs. $<75 \%$ ).

\section{DISCUSSION}

The prognostic impact of postinduction CS in the COG A3973 study has been previously reported (19). We now validate the utility of postinduction CS in an independent dataset, SIOPEN/ HR-NBL1. Both in SIOPEN/HR-NBL1 and as reported in COG A3973 (19), a postinduction CS of more than 2 was associated with an extremely poor outcome, with 5-y EFS of $16.4 \% \pm 4.2 \%$ (SIOPEN) and 10.5\% $\pm 10.0 \%$ (COG A3973), respectively. In contrast, patients with a postinduction CS of 2 or less had improved EFS in both SIOPEN/HR-NBL1 and COG A3973, with 5 -y EFS of $39.2 \% \pm 4.7 \%$ and $42.0 \% \pm 5.8 \%$, respectively (19). This finding suggests that patients with a CS of 2 or less after induction benefit from autologous stem cell transplantation and consolidation therapy but that patients with a higher CS may need alternative therapy to improve remission status.

COG A3973 and SIOPEN/HR-NBL1 treated a similar cohort of patients, specifically those with newly diagnosed, high-risk neuroblastoma $(3,22)$. Each trial shared a common backbone, with induction therapy, autologous stem cell transplantation, radiotherapy to the primary tumor site, and maintenance therapy with isotretinoin, with receipt of immunotherapy (chimeric antibody, ch14.18) in a small subset of patients $(<20 \%)$. Eligibility criteria approximated each other, though SIOPEN/HR-NBL1 included infants (<12 mo) with MYCN-amplified disease, and COG A3973 included infants $(<18 \mathrm{mo})$ with unfavorable biology $(M Y C N$ amplification, unfavorable histology, or a tumor DNA index of 1). There were distinct differences between the two trials, however. Induction consisted of 6 cycles of therapy, including 2 anthracyclineand 2 cisplatin-based cycles in COG A3973, compared with 8 induction cycles (at 10-d intervals, no anthracycline, 4 cycles of cisplatin) of Rapid COJEC administered in SIOPEN/HR-NBL1 $(3,22)$. Patients in SIOPEN/HR-NBL1 were randomized to receive either busulfan-melphalan or carboplatin-etoposide-melphalan for transplantation, with unpurged stem cells being the donor source. Patients in COG A3973 were randomized to receive purged or unpurged autologous grafts, with patients receiving carboplatinetoposide-melphalan for transplantation conditioning. A major difference was the timing of second-look surgery between the two

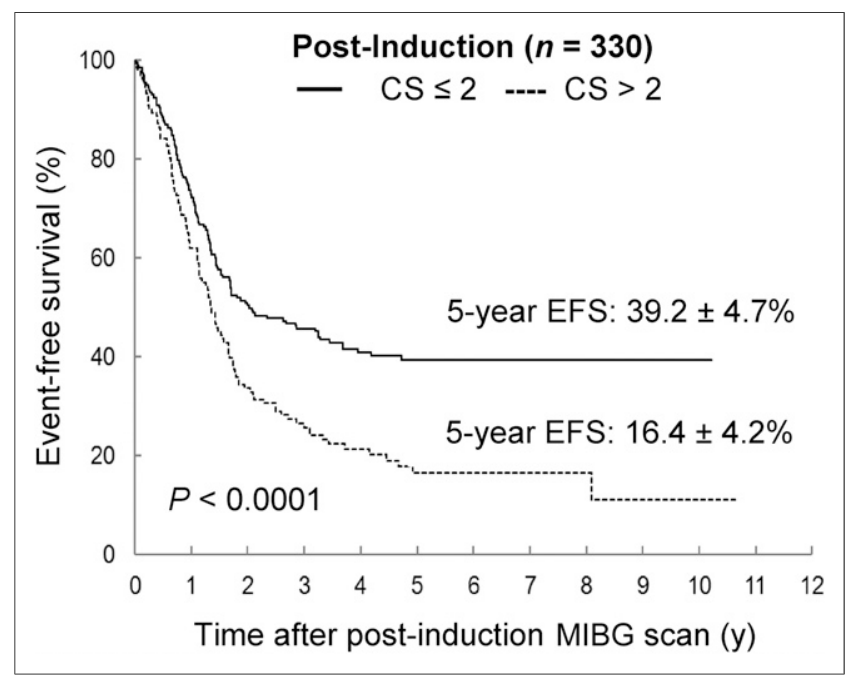

FIGURE 6. EFS by postinduction CS, using optimal cut point of 2: time to event starting from date of corresponding ${ }^{123}$ I-MIBG scan. 
studies. Whereas surgical resection was performed after induction in SIOPEN/HR-NBL1, patients treated in COG A3973 underwent surgical resection of their primary tumor before completion of induction therapy. In contrast to the COG A3973 cohort, more than 50\% of all patients in the SIOPEN study had ${ }^{123}$ I-MIBG-avid soft-tissue disease at the time of the postinduction ${ }^{123}$ I-MIBG scan, before their surgical resection. Despite these differences, our CS analyses came to the same conclusion in each trial, with a postinduction CS of more than 2 being associated with an inferior outcome.

The prognostic significance of CS at the time of initial diagnosis remains unclear. In SIOPEN/HR-NBL1, a cut point of 12 (at diagnosis) could differentiate patients who had a subsequent event from those who did not. In COG A3973, a higher CS at diagnosis was also associated with an inferior outcome, though the outcome difference did not reach statistical significance (19). Differences between the two induction regimens may help explain these findings, with a "modified N7" regimen being used in COG A3973 and a Rapid COJEC regimen being used in SIOPEN/HR-NBL1.

There were several limitations to our analysis. The conclusions were derived from a treatment protocol under which patients received a single transplant (busulfan-melphalan or carboplatinetoposide-melphalan) followed by local radiotherapy. The impact of postinduction CS in other scenarios, including receipt of a tandem transplant, is not yet established. The prognostic significance of postinduction CS in patients receiving immunotherapy is likewise unclear, with only 18\% (COG A3973) and 7\% (SIOPEN/HR-NBL1) of patients receiving immunotherapy in our CS analysis. In COG A3973, immunotherapy with ch14.18 was recommended but not mandatory. In SIOPEN/HR-NBL1, patients were assigned to receive immunotherapy in only the latter portion of the study, from 2007 onward. The impact of postinduction CS in large groups of patients who receive immunotherapy may become known on completion of current COG high-risk trials, including ANBL12P1 (NCT01798004).

$\mathrm{CS}$ is based on whole-body planar images. Many centers are currently performing SPECT imaging in addition to whole-body planar imaging. ${ }^{123}$ I-MIBG-avid lesions identified on SPECT imaging are not included in the CS unless they are also identified on whole-body planar imaging.

Two ${ }^{123}$ I-MIBG scoring methods currently exist, the CS and the SIOPEN score methodologies, with CS being used in COG studies and SIOPEN score being used in SIOPEN studies. A major difference between the two methodologies is the inclusion of softtissue scoring in the CS method, with the SIOPEN method being limited to skeletal disease. Whereas CS is a composite of skeletal $(90 \%)$ and soft-tissue $(10 \%)$ scores, the SIOPEN score is based primarily on skeletal scores. Whether the soft-tissue region should be included in CS is currently being examined in ongoing analyses of the COG and SIOPEN trials.

Our analysis is a testimony to the tremendous collaboration between investigators in two large, cooperative groups, COG and SIOPEN. Cross validation of each scoring method (CS and SIOPEN score) was performed, with COG investigators traveling to Vienna, Austria, to determine the CS on SIOPEN/HR-NBL1 scans and SIOPEN investigators traveling to the COG image repository (Quality Assurance Review Center) to determine the SIOPEN score on COG A3973 scans. The unique aspect of our collective work is that both scoring methods have been crossvalidated using the other's dataset, with the predictive value of the SIOPEN score method to be reported in a separate article. Standardized ${ }^{123} \mathrm{I}-\mathrm{MIBG}$ scores have now been incorporated into cooperative group neuroblastoma trials. Ultimately, a consensus scoring method will be developed by the COG and SIOPEN investigators for global use by the pediatric oncology community.

\section{CONCLUSION}

We have now confirmed the prognostic significance of the postinduction CS in an independent dataset, SIOPEN/HR-NBL1, with a postinduction CS of more than 2 being associated with inferior survival. Future work to develop a consensus scoring methodology between the COG and the SIOPEN groups is in progress.

\section{DISCLOSURE}

The study was supported in part by the Elaine and Leland Blatt Foundation; NIH grants U10CA180899, U10CA98543, and U10CA29511; the Alex Lemonade Foundation; the Dougherty Foundation; the Ben Towne Foundation; and the Children's Neuroblastoma Cancer Foundation. No other potential conflict of interest relevant to this article was reported.

\section{ACKNOWLEDGMENTS}

We thank Thomas J. FitzGerald, MD, and the staff at the Quality Assurance Review Center, especially Deirdre Logan, Sandy Kessel, and Fran Laurie, for their support of this project. We also thank the National Cancer Institute Pediatric and Adolescent Solid Tumor Steering Committee for their assistance.

\section{REFERENCES}

1. Matthay KK, Reynolds CP, Seeger RC, et al. Long-term results for children with high-risk neuroblastoma treated on a randomized trial of myeloablative therapy followed by 13-cis-retinoic acid: a children's oncology group study. J Clin Oncol. 2009;27:1007-1013.

2. Ladenstein R, Valteau-Couanet D, Brock P, et al. Randomized trial of prophylactic granulocyte colony-stimulating factor during rapid COJEC induction in pediatric patients with high-risk neuroblastoma: European SIOPEN/HR-NBL-1 study. J Clin Oncol. 2010;28:3516-3524.

3. Ladenstein R, Potschger U, Pearson AD, et al. Busulfan and melphalan versus carboplatin, etoposide, and melphalan as high-dose chemotherapy for high-risk neuroblastoma (SIOPEN/HR-NBL1): an international, randomised, multi-arm, open-label, phase 3 trial. Lancet Oncol. 2017;18:500-514.

4. Yu AL, Gilman AL, Ozkaynak MF, et al. Anti-GD2 antibody with GM-CSF, interleukin-2, and isotretinoin for neuroblastoma. N Engl J Med. 2010;363: 1324-1334.

5. London WB, Castel V, Monclair T, et al. Clinical and biologic features predictive of survival after relapse of neuroblastoma: a report from the International Neuroblastoma Risk Group project. J Clin Oncol. 2011;29:3286-3292.

6. Sisson JC, Frager MS, Valk TW, et al. Scintigraphic localization of pheochromocytoma. N Engl J Med. 1981;305:12-17.

7. Treuner J, Feine U, Niethammer D, et al. Scintigraphic imaging of neuroblastoma with [131-I]iodobenzylguanidine. Lancet. 1984;323:333-334.

8. Lumbroso JD, Guermazi F, Hartmann O, et al. Meta-iodobenzylguanidine (MIBG) scans in neuroblastoma: sensitivity and specificity, a review of 115 scans. Prog Clin Biol Res. 1988;271:689-705.

9. Shulkin BL, Shapiro B. Current concepts on the diagnostic use of MIBG in children. J Nucl Med. 1998;39:679-688.

10. Ady N, Zucker JM, Asselain B, et al. A new ${ }^{123}$ I-MIBG whole body scan scoring method: application to the prediction of the response of metastases to induction chemotherapy in stage IV neuroblastoma. Eur J Cancer. 1995;31A:256-261.

11. Perel Y, Conway J, Kletzel M, et al. Clinical impact and prognostic value of metaiodobenzylguanidine imaging in children with metastatic neuroblastoma. J Pediatr Hematol Oncol. 1999;21:13-18.

12. Suc A, Lumbroso J, Rubie H, et al. Metastatic neuroblastoma in children older than one year: prognostic significance of the initial metaiodobenzylguanidine scan and proposal for a scoring system. Cancer. 1996;77:805-811. 
13. Kushner BH, Yeh SD, Kramer K, et al. Impact of metaiodobenzylguanidine scintigraphy on assessing response of high-risk neuroblastoma to dose-intensive induction chemotherapy. J Clin Oncol. 2003;21:1082-1086.

14. Matthay KK, Edeline V, Lumbroso J, et al. Correlation of early metastatic response by ${ }^{123} \mathrm{I}$-metaiodobenzylguanidine scintigraphy with overall response and event-free survival in stage IV neuroblastoma. J Clin Oncol. 2003;21:2486-2491.

15. Messina JA, Cheng S-C, Franc B, et al. Evaluation of semi-quantitative scoring system for metaiodobenzylguanidine (MIBG) scans in patients with relapsed neuroblastoma. Pediatr Blood Cancer. 2006;47:865-874.

16. Katzenstein HM, Cohn S, Shore R, et al. Scintigraphic response by ${ }^{123}$ I-metaiodobenzylguanidine scan correlates with event-free survival in high-risk neuroblastoma. J Clin Oncol. 2004;22:3909-3915.

17. Schmidt M, Simon T, Hero B, et al. The prognostic impact of functional imaging with ${ }^{123}$ I-MIBG in patients with stage 4 neuroblastoma $>1$ year of age on a highrisk treatment protocol: results of the German Neuroblastoma Trial NB97. Eur J Cancer. 2008;44:1552-1558.

18. Decarolis B, Schneider C, Hero B, et al. Iodine-123 metaiodobenzylguanidine scintigraphy scoring allows prediction of outcome in patients with stage 4 neuroblastoma: results of the Cologne interscore comparison study. J Clin Oncol. 2013;31:944-951.

19. Yanik GA, Parisi MT, Shulkin BL, et al. Semiquantitative MIBG scoring as a prognostic indicator in patients with stage 4 neuroblastoma: a report from the Children's oncology group. J Nucl Med. 2013;54:541-548.

20. Lewington V, Lambert B, Poetschger U, et al. I-mIBG scintigraphy in neuroblastoma: development of a SIOPEN semi-quantitative reporting, method by an international panel. Eur J Nucl Med Mol Imaging. 2017;44:234-241.
21. Naranjo A, Parisi MT, Shulkin BL, et al. Comparison of ${ }^{123}$ I-metaiodobenzylguanidine (MIBG) and ${ }^{131}$ I-MIBG semi-quantitative scores in predicting survival in patients with stage 4 neuroblastoma: a report from the Children's Oncology Group. Pediatr Blood Cancer. 2011;56:1041-1045.

22. Kreissman SG, Seeger RC, Matthay KK, et al. Purged versus non-purged peripheral blood stem-cell transplantation for high-risk neuroblastoma (COG A3973): a randomised phase 3 trial. Lancet Oncol. 2013;14:999-1008.

23. Brodeur GM, Pritchard J, Berthold F, et al. Revisions of the international criteria for neuroblastoma diagnosis, staging, and response to treatment. J Clin Oncol. 1993;11:1466-1477.

24. Pearson AD, Pinkerton CR, Lewis IJ, Imeson J, Ellershaw C, Machin D. Highdose rapid and standard induction chemotherapy for patients aged over 1 year with stage 4 neuroblastoma: a randomised trial. Lancet Oncol. 2008;9:247256.

25. Veal GJ, Nguyen L, Paci A, et al. Busulfan pharmacokinetics following intravenous and oral dosing regimens in children receiving high-dose myeloablative chemotherapy for high-risk neuroblastoma as part of the SIOPEN/HR-NBL-1 trial. Eur J Cancer. 2012;48:3063-3072.

26. Youden WJ. Index for rating diagnostic tests. Cancer. 1950;3:32-35.

27. Pepe MS. The Statistical Evaluation of Medical Tests for Classification and Prediction. New York, NY: Oxford University Press; 2003.

28. Kaplan E, Meier P. Nonparametric estimation from incomplete observations. J Am Statist. 1958;53:457-481.

29. Peto R, Pike MC, Armitage P, et al. Design and analysis of randomized clinical trials requiring prolonged observation of each patient. II. analysis and examples. Br J Cancer. 1977;35:1-39. 